\title{
Self-consistent computation of electronic and optical properties of a single exciton in a spherical quantum dot via matrix diagonalization method
}

\author{
Mehmet Şahin, ${ }^{1, a)}$ Sedat Nizamoglu, ${ }^{2}$ A. Emre Kavruk, ${ }^{3}$ and Hilmi Volkan Demir ${ }^{4}$ \\ ${ }^{1}$ Department of Physics, Faculty of Sciences, Selçuk University, Kampüs 42075 Konya, Turkey \\ and Institute of Material Science and Nanotechnology, Bilkent University, 06800 Ankara, Turkey \\ ${ }^{2}$ Nanotechnology Research Center and Institute of Materials Science and Nanotechnology, \\ Bilkent University, 06800 Ankara, Turkey and Department of Electrical and Electronics Engineering, \\ Bilkent University, 06800 Ankara, Turkey \\ ${ }^{3}$ Department of Physics, Faculty of Sciences, Selçuk University, Kampüs 42075 Konya, Turkey \\ ${ }^{4}$ Nanotechnology Research Center and Institute of Materials Science and Nanotechnology, Bilkent University, \\ 06800 Ankara, Turkey; Department of Electrical and Electronics Engineering, Bilkent University, \\ 06800 Ankara, Turkey; and Department of Physics, Bilkent University, 06800 Ankara, Turkey
}

(Received 14 April 2009; accepted 15 June 2009; published online 21 August 2009)

In this study, we develop and demonstrate an efficient self-consistent calculation schema that computes the electronic structure and optical properties of a single exciton in a spherical quantum dot (QD) with an interacting pair of electron and hole wave functions. To observe modifications on bands, wave functions, and energies due to the attractive Coulomb potential, the full numeric matrix diagonalization technique is employed to determine sublevel energy eigenvalues and their wave functions in effective mass approximation. This treatment allows to observe that the conduction and valance band edges bend, that the electron and hole wave functions strongly localize in the QD, and that the excitonic energy level exhibits redshift. In our approach for the Coulomb term between electron and hole, the Poisson-Schrödinger equations are solved self-consistently in the Hartree approximation. Subsequently, exciton binding energies and associated optical properties are computed. The results are presented as a function of QD radii and photon energies. We conclude that all of these numerical results are in agreement with the experimental studies. (C) 2009 American Institute of Physics. [DOI: 10.1063/1.3197034]

\section{INTRODUCTION}

Recent developments in material synthesis and growth have made strong confinement of excitons possible in semiconductor low-dimensional structures [e.g., quantum wires, quantum dots (QDs) $]{ }^{1-3}$ In such strong confinement regime, the quantum mechanical effects dominate with diminishing size of these nanostructures. These effects provide different advantages in designing new generation electronic and optical devices including QD infrared photodetectors, light emitting diodes, and optical memories. ${ }^{4-6}$ Because the semiconductor quantum structures have a great potential for device applications, their exciton mechanisms are widely investigated both theoretically and experimentally in condensed matter physics and applied sciences. ${ }^{7-18}$

The electronic structure calculations of excitons are key to understanding the resulting optical properties and designing functional nanodevices. The first theoretical study related to an exciton in a microsphere was reported by Efros and Efros. ${ }^{19}$ Subsequently, numerous studies have been reported using different methods and approximations. ${ }^{20-25}$ In most of these studies the description of the quantum confinement is based on the framework of the effective mass approximation (EMA). This is a simple but a useful approximation for electronic structure calculations. ${ }^{9,14,17,26}$ In strong confinement

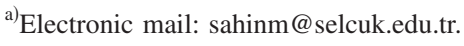

regimes, EMA has been shown to be sufficient for understanding electronic structure of these low-dimensional systems. $12,27,28$

In an exciton problem, proper handling and calculation of the Coulomb interaction term between electron and hole is known to be complicated. In most of the studies especially in strong confinement regime [i.e., dot radius $\left(R_{\text {dot }}\right) \ll$ exciton Bohr radius $\left.\left(a_{\mathrm{exc}}\right)\right]$, this Coulomb term is completely ignored comparing with the kinetic energy of the electron and hole in the calculations. ${ }^{16,24,29,30}$ In some cases, although the Coulomb term is considered as a perturbation and the first order energy modification is performed, the wave function is not modified. ${ }^{28,31}$ In these cases, although the energy levels are calculated with sufficient precision, computation of optical properties (oscillator strength, optical transitions, etc.) is not accurate enough as a result of the noninteracting electron and hole wave functions assumed in the formalism. To address this problem, different from the previous studies, in the present work the effect of the Coulomb potential between electron and hole on the wave function is included in our calculations.

For this purpose in this study, we introduce an efficient computation schema that relies on full numeric matrix diagonalization method in the EMA using Hartree treatment. In this context, we compute the electronic structure and the resulting optical properties of an exciton in a spherical QD effectively including the Coulomb term in all calculations. To this end, the Poisson-Schrödinger equations are solved 
self-consistently in the Hartree approximation to determine the eigenenergies and the corresponding eigenfunctions of the exciton in a QD. As a result, the application area of EMA is to be expanded to generic exciton problems with Hartree approximation in this study.

This paper is organized as follows. In Sec. II, the calculation method is presented. Subsequently, results and discussions are provided in Sec. III. In Sec. IV, conclusions are presented.

\section{METHOD}

In this study, a spherically symmetric QD with radius $R_{\text {dot }}$, which is embedded in a bulk semiconductor, is considered. The EMA and BenDaniel-Duke boundary conditions are used for self-consistent calculations. In the EMA, the Schrödinger equation of an exciton for a spherical QD is given as

$$
\begin{aligned}
& {\left[-\frac{\hbar^{2}}{2} \vec{\nabla}_{r}\left(\frac{1}{m_{e}^{*}(r)} \vec{\nabla}_{r}\right)-\frac{\hbar^{2}}{2} \vec{\nabla}_{r}\left(\frac{1}{m_{h}^{*}(r)} \vec{\nabla}_{r}\right)-\frac{e^{2}}{\kappa\left|\vec{r}_{e}-\vec{r}_{h}\right|}\right.} \\
& \left.\quad+\frac{\ell(\ell+1) \hbar^{2}}{2 m_{e}^{*}(r) r^{2}}+\frac{\ell(\ell+1) \hbar^{2}}{2 m_{h}^{*}(r) r^{2}}+V_{e}+V_{h}\right] R_{n, \ell}^{\mathrm{exc}}(r) \\
& =\varepsilon_{n, \ell} R_{n, \ell}^{\mathrm{exc}}(r) .
\end{aligned}
$$

Here $\hbar$ is reduced Planck constant, $m_{e, h}^{*}(r)$ is the positiondependent electron's and hole's effective masses, $e$ is the unit electronic charge, $\ell$ is the angular momentum quantum number, $V_{e, h}$ is the finite confining potential of electron and hole, $\varepsilon_{n, \ell}$ is the exciton energy eigenvalue, and $R_{n, \ell}^{\text {exc }}(r)$ is the radial wave function of the exciton.

It is impossible to solve this equation analytically and hence numerical calculations are indispensable. For this purpose, we express the Eq. (1) in two pieces separately for electron and hole using Hartree approximation as follows:

$$
\begin{aligned}
& {\left[-\frac{\hbar^{2}}{2} \vec{\nabla}_{r}\left(\frac{1}{m_{e}^{*}(r)} \vec{\nabla}_{r}\right)-e \Phi_{h}+\frac{\ell(\ell+1) \hbar^{2}}{2 m_{e}^{*}(r) r^{2}}+V_{e}\right] R_{n, \ell}^{\text {elec }}(r)} \\
& =\varepsilon_{n, \ell}^{\text {elec }} R_{n, \ell}^{\text {elec }}(r),
\end{aligned}
$$

and

$$
\begin{aligned}
& {\left[-\frac{\hbar^{2}}{2} \vec{\nabla}_{r}\left(\frac{1}{m_{h}^{*}(r)} \vec{\nabla}_{r}\right)-e \Phi_{e}+\frac{\ell(\ell+1) \hbar^{2}}{2 m_{h}^{*}(r) r^{2}}+V_{h}\right] R_{n, \ell}^{\text {hole }}(r)} \\
& =\varepsilon_{n, \ell}^{\text {hole }} R_{n, \ell}^{\text {hole }}(r) .
\end{aligned}
$$

In the last two equations, the first terms are kinetic energy terms of electron and hole, respectively. The second terms represent the electrostatic Coulomb potential between electron and hole. Here $\Phi_{e}$ and $\Phi_{h}$ are the Hartree potential of the electron and hole, respectively. The Hartree approximation used in the formalism supposes that one particle (electron or hole) moves in a mean potential field created by other particle(s) in a many-particle system. ${ }^{32}$ Although this model is quite simple, its results are accurate for the understanding of the resultant properties of a many-particle system. The third term in Eqs. (2) and (3) comes from the angular momentum of electron and hole. $\varepsilon_{n, \ell}^{\text {elec }}$ and $\varepsilon_{n, \ell}^{\text {hole }}$ are the respective electron and hole energy eigenvalues; $R_{n, \ell}^{\text {elec }}(r)$ and $R_{n, \ell}^{\text {hole }}(r)$ are the radial wave functions of the electron and hole, respectively. In this schema, we consider that the electron moves in the electrostatic hole potential and similarly the hole moves in the electrostatic electron potential. The electron and hole potentials are calculated from the Poisson equation by means of

$$
\nabla^{2} \Phi_{e}=\frac{e \rho_{e}(r)}{\kappa(r)}
$$

and

$$
\nabla^{2} \Phi_{h}=-\frac{e \rho_{h}(r)}{\kappa(r)}
$$

where $\rho_{e}(r)$ is the electron density, $\rho_{h}(r)$ is the hole density, and $\kappa(r)$ is the dielectric constant of the structure. The electron and hole densities are

$$
\rho_{e}(r)=\frac{1}{4 \pi} \sum_{\ell=0}^{p} 2(2 \ell+1) \sum_{n=1}^{n p}\left|R_{n, \ell}^{\mathrm{elec}}(r)\right|^{2}+\frac{1}{4 \pi} q\left|R_{n q, \ell q}^{\mathrm{elec}}(r)\right|^{2},
$$

and

$$
\rho_{h}(r)=\frac{1}{4 \pi} \sum_{\ell=0}^{p} 2(2 \ell+1) \sum_{n=1}^{n p}\left|R_{n, \ell}^{\text {hole }}(r)\right|^{2}+\frac{1}{4 \pi} q\left|R_{n q, \ell q}^{\text {hole }}(r)\right|^{2} .
$$

Here $2(2 \ell+1)$ is the spin and magnetic degeneracies; $p$ and $n p$ are the angular momentum quantum number and the principle quantum number of the fully occupied states, respectively; $q$ is the number of remaining electrons (or holes) in the last state; $n q$ and $\ell q$ are the principle quantum number and angular momentum quantum number of the last state, respectively. For a single exciton the maximum electron and hole number are $N=1$ and so only $1 s$ shell of conduction and valance bands is occupied with the electron and hole.

The matrix diagonalization technique is used for the determination of the single particle eigenenergies and corresponding eigenfunctions. For this purpose, Hamiltonian operator is discretized using the finite differences on a uniform radial mesh in 1 dimension (1D), then Eqs. (2) and (3) are then reduced into a matrix eigenvalue equation. In the discritization $\Delta r$ width between two mesh points are set as 0.005. Eigenvalues and eigenvectors of this matrix equation are determined by EISPACK subroutine.

In the first step, Eqs. (2) and (3) are diagonalized without the Hartree potential to determine the single electron and hole energy levels and their corresponding wave functions. Consequently, the energy levels and wave functions of noninteracting electron and hole are computed. In the second step, the electron and hole radial wave functions that are determined in the first step are used in Eqs. (6) and (7) to determine the electron and hole electronic charge densities. Afterward, the calculated charge densities are used in Poisson Eqs. (4) and (5), and the electron and hole Hartree potential are calculated. For the calculation of Hartree potential, the finite difference technique with Gauss elimination method is used. To perform a self-consistent calculation, the Hartree potential of hole and electron is substituted into Eqs. 
TABLE I. The material parameters used in the calculations.

\begin{tabular}{lllccc}
\hline \hline Material & $m_{e}^{* a}$ & $m_{h}^{* \mathrm{~b}}$ & $\kappa^{\mathrm{a}}$ & $\begin{array}{c}E_{g} \\
(\mathrm{eV})^{\mathrm{a}}\end{array}$ & $\chi^{\mathrm{a}}$ \\
\hline CdSe & $0.13 m_{0}$ & $0.45 m_{0}$ & 9.3 & 1.75 & 4.95 \\
ZnS & $0.28 m_{0}$ & $0.49 m_{0}$ & 8.1 & 3.75 & 3.90 \\
\hline
\end{tabular}

${ }^{\mathrm{a}}$ from Ref. 33.

${ }^{\mathrm{b}}$ from Ref. 7.

(2) and (3), respectively, and this process is continued until they converge implying that the interacting single electron and hole energy values and wave functions had been determined.

The total energy (exciton transition energy) are computed by means of

$$
E_{\mathrm{exc}}=E_{g}+\varepsilon_{\mathrm{elec}}+\varepsilon_{\mathrm{hole}}-\varepsilon_{\mathrm{coul}} .
$$

Here $E_{g}$ is the band gap energy of the bulk semiconductor material, $\varepsilon_{\text {elec }}$ and $\varepsilon_{\text {hole }}$ are the respective discrete electron and hole energy levels due to the quantum confinement effect and $\varepsilon_{\text {coul }}$ is the Coulomb energy between electron and hole. In the computation of total energy, since the Coulomb term is already considered in self-consistent calculation of both the electron and hole energies, the Coulomb term is double counted. Therefore, the Coulomb term must be subtracted to correct the total energy. The Coulomb energy can thus be calculated as

$$
\varepsilon_{\text {coul }}=-\frac{\left(\varepsilon_{\text {elec }}^{0}-\varepsilon_{\text {elec }}+\varepsilon_{\text {hole }}^{0}-\varepsilon_{\text {hole }}\right)}{2} .
$$

Here $\varepsilon_{\text {elec }}^{0}$ and $\varepsilon_{\text {hole }}^{0}$ are the noninteracting electron and hole energies calculated in the first step, respectively. $\varepsilon_{\text {elec }}$ and $\varepsilon_{\text {hole }}$ are the interacting electron and hole energies determined by the self-consistent computation in the last step.

\section{RESULTS AND DISCUSSIONS}

In this study, we consider wurtzite type CdSe core and $\mathrm{CdSe} / \mathrm{ZnS}$ core/shell nanocrystal QD structures. The atomic units have been used throughout the calculations, where Planck constant $\hbar=1$, the electronic charge $e=1$, and the electron mass $m_{0}=1$. By using the material parameters presented in Table I, the effective Bohr radius is $a_{0}^{*}=48.78 \AA$ and the effective Rydberg energy is $R_{y}^{*}=15.86 \mathrm{meV}$ for $\mathrm{CdSe}$ core QD. The exact conduction and valence band offsets of $\mathrm{CdSe} / \mathrm{ZnS}$ core/shell QD are not available in the literature and, therefore, the band offsets are determined by using electron affinity and band gap energies of materials $\mathrm{CdSe}$ and $\mathrm{ZnS}$ as summarized in Table I. Figure 1 shows the schematic representation of the band structure. The conduction band offset, which is electron confining potential, is calculated by $\Delta E_{C}=\chi_{1}-\chi_{2}$. Similarly, the hole confining potential is determined by $\Delta E_{V}=\Delta E_{g}-\Delta E_{C}$. As a result, the confining potential values are taken as $V_{e}=1.05 \mathrm{eV}$ and $V_{h}$ $=0.95 \mathrm{eV}$.

Our calculation schema has an iterative and selfconsistent nature. In Fig. 2 the energy values of CdSe core QD embedded in a glass are plotted as a function of iteration (in our calculation for CdSe core QD the confining potential

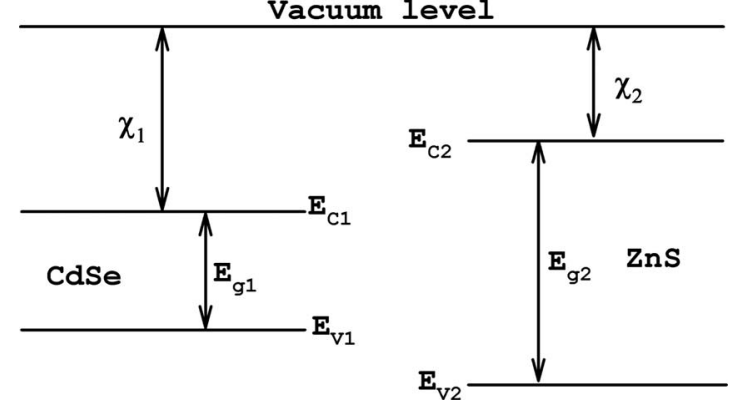

FIG. 1. Schematic energy band representation of CdSe/ZnS.

is taken as infinite). Here the zeroth iteration is the initial energy values of electron and hole for the case without Coulomb interaction. In the next iterations, the Coulomb term between electron and hole is included in the calculations. As seen from the figure, after a few iteration the computation quickly converges. The central processor unit (CPU) time for four iterations is approximately $12 \mathrm{~s}$ in an Intel Pentium-D processor. This makes our computational approach efficient. When we compare the energy of the zeroth iteration with the converged one, we observe that the electron and hole energies with and without Coulomb interaction are quite large because the attractive Coulomb potential between electron and hole pulls down the single electron and hole energies. In some of the excitonic studies, ${ }^{16,24,29,30}$ the Coulomb term effect on the energies is omitted; however, as showcased in the figure, in fact this contribution is not at a negligible level.

Because of the attractive Coulomb potential it is expected that the attractive Coulomb potential bends the conduction and valance band edges and also affects the electron and hole wave functions. In Fig. 3 these effects are clearly evident for $\mathrm{CdSe} / \mathrm{ZnS}$ core/shell QD with $R_{\mathrm{dot}}=2.2 \mathrm{~nm}$. As shown in the figure, the attractive Coulomb potential between electron and hole bends the conduction and valance band edges, and the bendings of the conduction and valance bands are approximately at the same level. Furthermore, in Fig. 3, the normalized wave functions are also depicted. The wave functions of the electron and hole are drastically af-

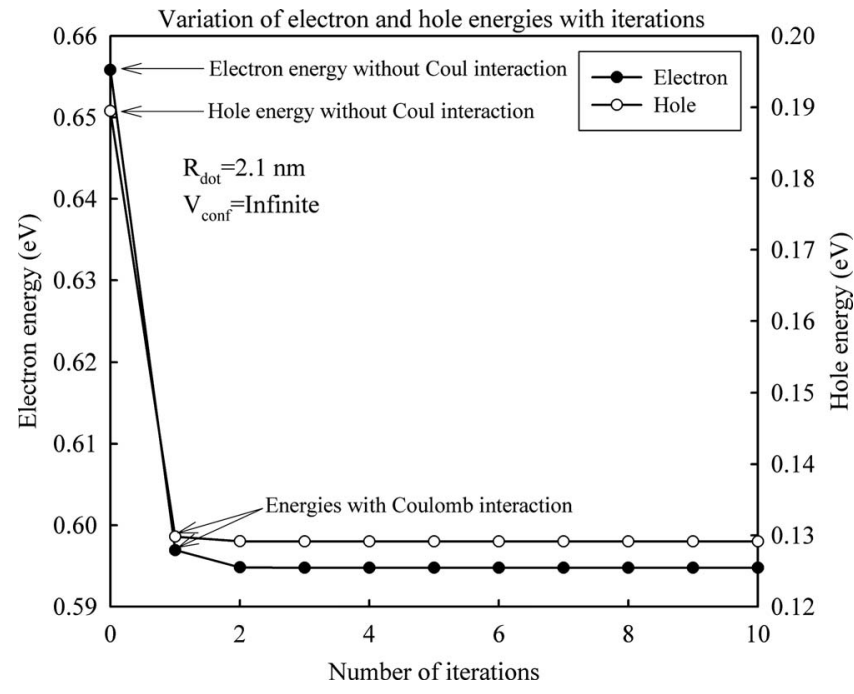

FIG. 2. Evolution of the electron and hole energies with iterations. 

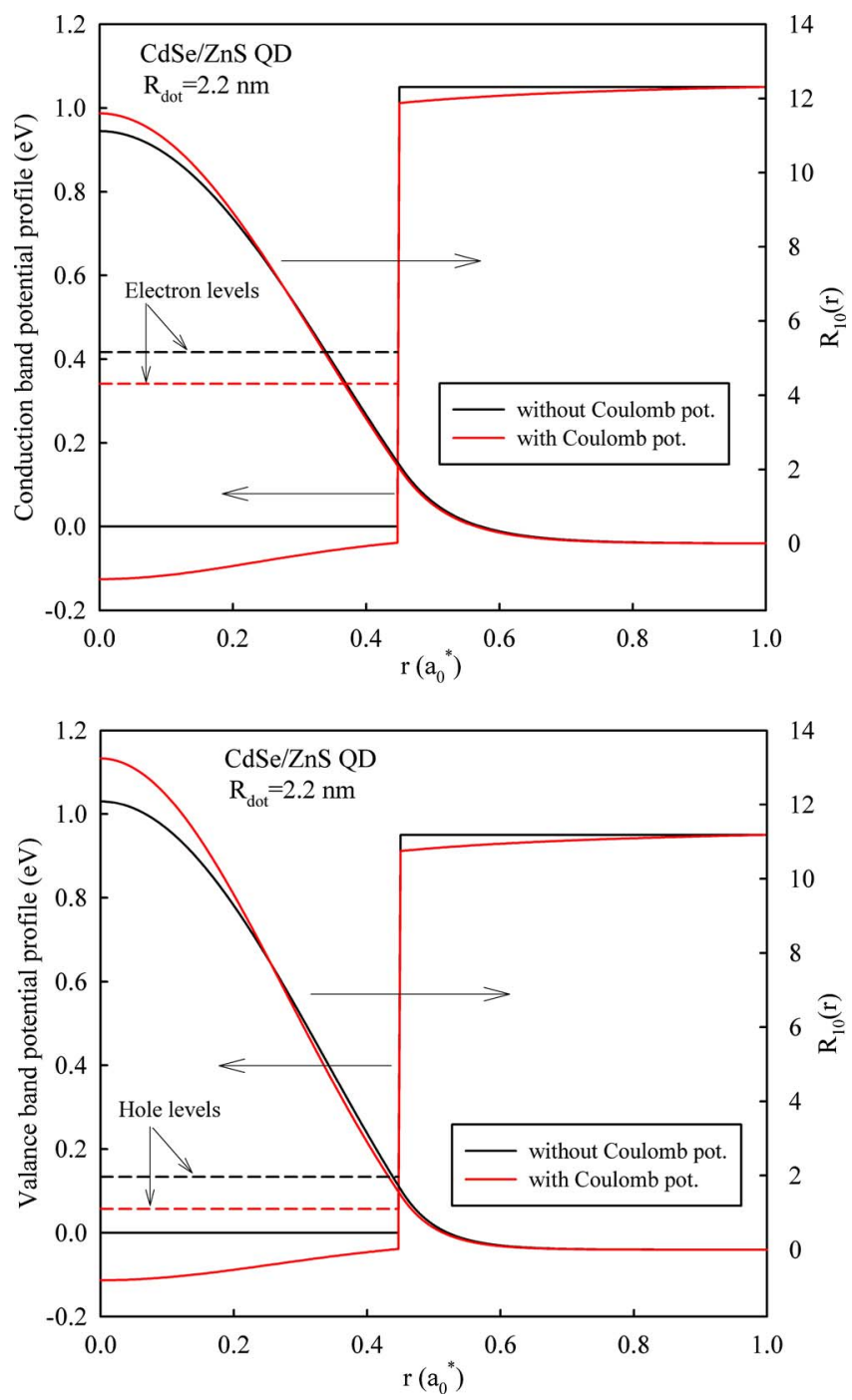

FIG. 3. (Color online) Self-consistent electronic structure of CdSe/ZnS core/ shell QD, band profile, energy values, and corresponding wave functions for electron (top panel) and for hole (bottom panel).

fected by the Coulomb term. Although the effect of Coulomb term between electron and hole is not taken into consideration in most of the studies, we observe that the interacting nature of the electron and hole significiantly affects the wave functions. In some previous studies, ${ }^{28,31}$ even though the first order perturbation is employed on the energies, the resulting wave functions are not modified at all. The effect of the Coulomb term on the wave functions may play on important role on the optical property calculations of low dimensional excitonic structures. Therefore, the consideration of the Coulomb term effect on both energy levels and their wave functions in optical properties studies of excitons is of critical importance.

By using our calculation scheme we also investigate the optical absorption coefficient of QDs. The general form of the exciton absorption coefficient of a single QD is given by

$$
\alpha(\hbar \omega) \propto \sum_{i} f_{i} \delta_{i}\left(\hbar \omega-E_{\mathrm{exc}}^{i}\right)
$$

where $f_{i}$ is the oscillator strength, $\delta_{i}$ is the line-shape function, $E_{\text {exc }}$ is the exciton transition energy, and $\hbar \omega$ is the in-
TABLE II. Comparison of theoretical and experimental results for bare CdSe QD embedded in a glass matrix.

\begin{tabular}{ccc}
\hline \hline $\begin{array}{c}R_{\text {dot }} \\
(\mathrm{nm})\end{array}$ & $\begin{array}{c}\text { Energy }(\exp )^{\mathrm{a}} \\
(\mathrm{eV})\end{array}$ & $\begin{array}{c}\text { Energy (theo })^{\mathrm{b}} \\
(\mathrm{eV})\end{array}$ \\
\hline 2.10 & 2.55 & 2.53 \\
2.60 & 2.27 & 2.25 \\
3.80 & 2.08 & 1.98 \\
\hline
\end{tabular}

${ }^{a}$ from Ref. 13.

b this study.

cident photon energy. The sum is over all the possible transitions. For the absorption band edge, only $1 s-1 s$ transition is taken into consideration. The oscillator strength for this optical transitions is calculated by means of ${ }^{34}$

$$
f=\frac{E_{p}}{2 E_{\mathrm{exc}}}\left|\int d^{3} r \psi_{e}(r) \psi_{h}(r)\right|^{2},
$$

where $E_{p}$ is the Kane energy, which is $21 \mathrm{eV}$ for CdSe. ${ }^{35}$ The electron and hole wave functions $\left[\psi_{e}(r)\right.$ and $\left.\psi_{h}(r)\right]$ are determined by the multiplication of the radial wave function determined by Eqs. (2) and (3) with the spherical harmonics [i.e., $\left.R_{n, \ell}(r) Y_{\ell, m}(\theta, \varphi)\right]$. We consider a homogeneous line broadening and select a hyperbolic secant type function for the line-shape function as

$$
\delta=\frac{1}{\pi \hbar \Gamma} \sec h\left(\frac{E_{\mathrm{exc}}-\hbar \omega}{\hbar \Gamma}\right) .
$$

The line-shape broadening factor $\hbar \Gamma$ is taken to be $30 \mathrm{meV}$.

The exciton transition in a bare QD embedded in a glass matrix, for example $\mathrm{CdSe}$, is still studied widely. Therefore the electronic structure calculation of this kind of QDs is important for the understanding of their optical properties. First, we applied our calculation schema to a bare CdSe QD embedded in a glass matrix. In this calculation, the confining potential is assumed to be infinite because the band gap of glass is about $7 \mathrm{eV}$. After electronic structure calculation of $\mathrm{CdSe}$ structure, the optical properties calculation procedure described above is followed for exciton transition. The results are presented in comparison with the experimental ones in Table II. The experimental results are taken from Ref. 13. We see that our $(1 s-1 s)$ absorption transition calculations are in agreement with the experimental results especially in strong confinement region. On the other hand, a shift of around $95 \mathrm{meV}$ is observed between the theoretical and experimental results for dot radius $R_{\mathrm{dot}}=3.8 \mathrm{~nm}$. This is attributed to the dielectric mismatch of the dot and the barrier materials, ${ }^{1,2}$ which becomes more dominant at large QD.

The exciton transition calculation of a $\mathrm{CdSe} / \mathrm{ZnS}$ core/ shell QD is also important for technological applications. Therefore, we calculate the electronic structure and optical properties of this QD using the self-consistent computation schema. We calculate the $(1 s-1 s)$ absorption transition of $\mathrm{CdSe} / \mathrm{ZnS}$ core/shell QDs with different radii including 1.7, $2.2,2.5$, and $2.9 \mathrm{~nm}$. In this calculation the thickness of the $\mathrm{ZnS}$ layer is taken to be $3 \mathrm{ML}$ (monolayers), for which $1 \mathrm{ML}$ is approximately $0.5 \mathrm{~nm}^{7}$ After the electronic structure calculation, the same procedure explained above is applied for 


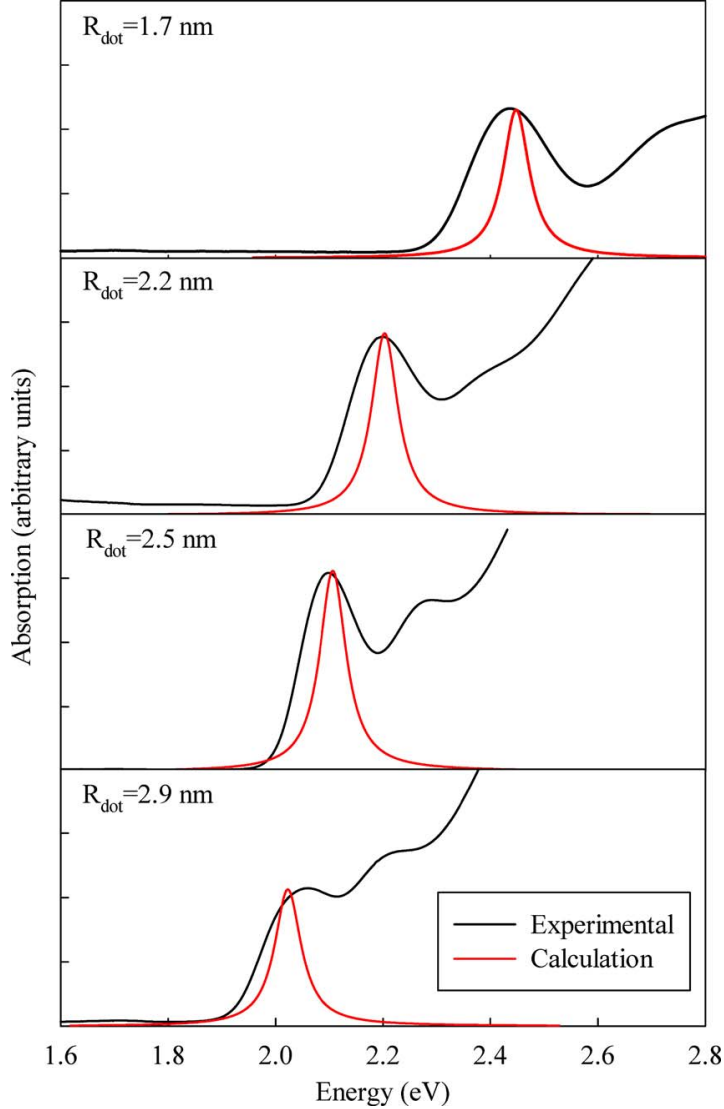

FIG. 4. (Color online) Absorption spectra of CdSe/ZnS core/shell QD. $R_{\mathrm{dot}}$ means core radius.

exciton transition. For comparison, we measure the absorption spectra of the same QDs in toluene. The experimental measurements are performed using $\mathrm{CdSe} / \mathrm{ZnS}$ core/shell QDs produced by Evident technologies ${ }^{36}$ with Varian spectrophotometer. In Fig. 4, our calculated and measured results are presented and they are in agreement with each other, although some numerical results diverge slightly from the experimental data. Because the transition energies strongly depend on the thickness of the shell, this minor difference is considered to stem from the fact that $\mathrm{ZnS}$ barrier is not necessarily exactly $3 \mathrm{ML}$ thick. The other possible factor can be the dielectric mismatch of the core, shell, and solution materials. If the image charge correction is taken into account in the calculations, this difference between the theoretical and experimental results may be reduced.

\section{CONCLUSION}

In this study, the electronic structure and optical properties of a single exciton confined in a spherical QD are selfconsistently computed in an EMA. The effect of Coulomb term between electron and hole both on energy levels and wave functions is considered in the calculations. It is observed that the results are in a good agreement with the experimental data both for bare $\mathrm{CdSe}$ core and $\mathrm{CdSe} / \mathrm{ZnS}$ core/ shell QDs. In addition to the computation of the energy level and wave function in an interacting electron and hole picture, the conduction and valance band bending is also calculated in this calculation procedure, which is not possible with other methods including variational approach and perturbation theory. Therefore, this efficient computation schema offers benefits in handling the Coulomb interaction term and provides the ability to observe the modification on the band, wave functions, and energy levels due to the Coulomb potential.

\section{ACKNOWLEDGMENTS}

This work is partially supported by Selçuk University BAP office. This work is in part supported by ESF-EURYI, TUBITAK EEEAG Grant Nos. 106E020, 107E088, 107E297, and 109E002 and TUBA-GEBIP.

${ }^{1}$ L. Banyai and S. W. Koch, Semiconductor Quantum Dots (World Scientific, Singapore, 1993).

${ }^{2}$ D. Bimberg, M. Grundmann, and N. N. Ledentsov, Quantum Dot Heterostructures (Wiley, Chichester, 1999).

${ }^{3}$ Y. Z. Hu, S. W. Koch, and N. Peyghambarian, J. Lumin. 70, 185 (1996).

${ }^{4}$ S. Nizamoglu, T. Ozel, E. Sari, and H. V. Demir, Nanotechnology 18, 065709 (2007).

${ }^{5}$ S. Nizamoglu and H. V. Demir, Nanotechnology 18, 405702 (2007).

${ }^{6}$ H. C. Liu, Opto-Electron. Rev. 11, 1 (2003).

${ }^{7}$ S. Nizamoglu and H. V. Demir, Opt. Express 16, 3515 (2008).

${ }^{8}$ C. Bulutay, Phys. Rev. B 76, 205321 (2007).

${ }^{9}$ L. P. Balet, S. A. Ivanov, A. Piryatinski, M. Achermann, and V. I. Klimov, Nano Lett. 4, 1485 (2004).

${ }^{10}$ D. J. Norris and M. G. Bawendi, Phys. Rev. B 53, 16338 (1996).

${ }^{11}$ G. A. Narvaez, G. Bester, and A. Zunger, Phys. Rev. B 72, 245318 (2005).

${ }^{12}$ Z. M. Schultz and J. M. Essick, Am. J. Phys. 76, 241 (2008).

${ }^{13}$ A. I. Ekimov, F. Hache, M. C. Schanne-Klein, D. Ricard, C. Flytzanis, I. A. Kudryavtsev, T. V. Yazeva, A. V. Rodina, and Al. L. Efros, J. Opt. Soc. Am. B 10, 100 (1993).

${ }^{14}$ U. Woggon, Optical Properties of Semiconductor Quantum Dots (Springer-Verlag, Berlin, Heidelberg, 1997).

${ }^{15}$ S. Gaponenko, Optical Properties of Semiconductor Nanocrystals (Cambridge University Press, Cambridge, England, 1998).

${ }^{16}$ U. Bockelmann and G. Bastard, Phys. Rev. B 45, 1688 (1992).

${ }^{17}$ S.-S. Kim, S.-K. Hong, and K.-H. Yeon, Phys. Rev. B 76, 115322 (2007).

${ }^{18}$ V. I. Klimov, J. A. McGuire, R. D. Schaller, and V. I. Rupasov, Phys. Rev. B 77, 195324 (2008).

${ }^{19}$ Al. L. Efros and A. L. Efros, Sov. Phys. Semicond. 16, 772 (1982).

${ }^{20}$ V. A. Shuvayev, L. I. Deych, I. V. Ponomarev, and A. A. Lisyansky, Superlattices Microstruct. 40, 77 (2006).

${ }^{21}$ L.-W. Wang and A. Zunger, Phys. Rev. B 53, 9579 (1996).

${ }^{22}$ T. Takagahara, Phys. Rev. B 39, 10206 (1989).

${ }^{23}$ L. Banyai, Y. Z. Hu, M. Lindberg, and S. W. Koch, Phys. Rev. B 38, 8142 (1988).

${ }^{24}$ Y. Kayanuma, Solid State Commun. 59, 405 (1986).

${ }^{25}$ U. E. H. Laheld and G. T. Einevoll, Phys. Rev. B 55, 5184 (1997).

${ }^{26}$ H. Gotoh and H. Ando, J. Appl. Phys. 82, 1667 (1997).

${ }^{27}$ T. Uozumi, Y. Kayanuma, K. Yamanaka, K. Edamatsu, and T. Itoh, Phys. Rev. B 59, 9826 (1999).

${ }^{28}$ V. I. Klimov, S. A. Ivanov, J. Nanda, M. Achermann, I. Bezel, J. A. McGuire, and A. Piryatinski, Nature (London) 447, 441 (2007).

${ }^{29}$ V. A. Harutyunyan, Physica E (Amsterdam) 39, 37 (2007).

${ }^{30}$ M. S. Atoyan, E. M. Kazaryan, and H. A. Sarkisyan, Physica E (Amsterdam) 22, 860 (2004).

${ }^{31}$ A. Piryatinski, S. A. Ivanov, S. Tretiak, and V. I. Klimov, Nano Lett. 7, 108 (2007)

${ }^{32}$ E. A. Johson, in Low Dimensional Semiconductor Structures, edited by K. Barnham and D. Vvedensky (Cambridge University Press, Cambridge, England, 2001), p. 79.

${ }^{33} \mathrm{~S}$. Adachi, Properties of Group IV, III-V and II-VI Semiconductors (Wiley, England, 2005).

${ }^{34}$ J. H. Davies, The Physics of Low-Dimensional Semiconductors: An Introduction (Cambridge University Press, Cambridge, England, 1996).

${ }^{35}$ E. W. Van Stryland, M. A. Woodall, H. Vanherzeele, and M. J. Soileau, Opt. Lett. 10, 490 (1985).

${ }^{36} \mathrm{http}: / / \mathrm{www}$. evidenttech.com/products/evidots.html 